\title{
Toward Automated Multiparty Privacy Conflict Detection
}

\author{
Haoti Zhong \\ Penn State University, Department of \\ Electrical Engineering \\ hzz113@psu.edu
}

\author{
Anna Squicciarini \\ Penn State University, College of \\ Information Sciences and Technology \\ acs20@psu.edu \\ David Miller \\ Penn State University, Department of \\ Electrical Engineering \\ djmiller@engr.psu.edu
}

\begin{abstract}
In an effort to support users' decision making process in regards to shared and co-managed online images, in this paper we present a novel model to early detect images which may be subject to possible conflicting access control decisions. We present a groupbased stochastic model able to identify potential privacy conflicts among multiple stakeholders of an image. We discuss experiments on a dataset of over 3000 online images. Our approach outperforms all baselines, even the strong ones based on a Convolutional Neural Network architecture.
\end{abstract}

\section{CCS CONCEPTS}

- Security and privacy $\rightarrow$ Privacy protections;

\section{KEYWORDS}

Privacy, Personalized models, Multi-Party Access Control

\section{ACM Reference Format:}

Haoti Zhong, Anna Squicciarini, and David Miller. 2018. Toward Automated Multiparty Privacy Conflict Detection. In The 27th ACM International Conference on Information and Knowledge Management (CIKM '18), October 22-26, 2018, Torino, Italy. ACM, New York, NY, USA, 4 pages. https://doi.org/10.1145/3269206.3269329

\section{INTRODUCTION}

Content sharing in online settings is now one of the most common activities of Internet users, who often have to make access control or privacy-related decisions that impact themselves and other content stakeholders. These decisions involve manual negotiations, often resulting in unsatisfactory outcomes, and may lead to avoidance, aggression or even withdrawal $[4,6]$. Issues related to joint access decisions, or multi-party access control, have recently emerged as an important and non-trivial challenge to address in online sharing, especially when lay users are asked to make a large number of decisions, with a general rule for addressing possible conflicts among image co-owners (or stakeholders) not easily derived [6, 7]. Toward addressing these issues, recent work has proposed various mechanisms to resolve multi-party privacy conflicts in social media (e.g. [4]), each offering different degrees of automation and users' direct involvement. However, to the best of our knowledge, the emphasis

Permission to make digital or hard copies of all or part of this work for personal or classroom use is granted without fee provided that copies are not made or distributed for profit or commercial advantage and that copies bear this notice and the full citation on the first page. Copyrights for components of this work owned by others than ACM must be honored. Abstracting with credit is permitted. To copy otherwise, or republish, to post on servers or to redistribute to lists, requires prior specific permission and/or a fee. Request permissions from permissions@acm.org.

CIKM '18, October 22-26, 2018, Torino, Italy

(C) 2018 Association for Computing Machinery.

ACM ISBN 978-1-4503-6014-2/18/10.

https://doi.org/10.1145/3269206.3269329

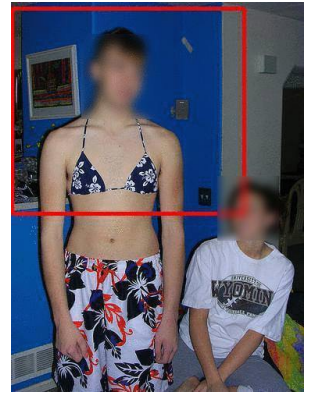

Figure 1: Example of images with a region of focus and two distinct stakeholders.

so far has been on managing conflicts, rather than detecting them. In this paper, we present the first automated method that is able to identify potential privacy conflicts for a given image, according to the involved stakeholders and their relationship

The ultimate goal is to provide an accurate early warning system for identifying conflicting privacy preferences among image stakeholders. We define a policy conflict as follows. For a given image, assume there is one uploader and up to $K$ tagged users. Users may be connected with one another according to a social connection, e.g. first degree relationship (friends, colleagues) or secondary degree (e.g. social circle). Assume that users are to choose among an ordinal set of "privacy" or image sharing options, ordered by degree of strictness. We say a conflict exists when a tagged user has a stricter privacy preference than the uploader. We assume a scale of sharing settings ranked from from 1 to 4 , with level 1 meaning clearly public, 2 public, 3 private, and 4 clearly private ${ }^{1}$.

Our approach to predict conflicts is based on a group-based model for image privacy classification, that accounts for multiple stakeholders at the same time. We design our solution based on the following considerations, informed by recent works in the area of multi-party access control (e.g. [4, 7, 9]). We hypothesize that users will focus primarily on the portions of the content that mostly resonate to them, e.g the uploader will likely focus on the whole image, and the user(s) being tagged on the portions of the image where they are portrayed (or their family/children). For ease of explanation, we refer to this portion of content within an image as a "focused region". Clearly, as users observe a single image, the content surrounding their region of focus will also matter - but perhaps with lesser importance. Thus, in the case of a "tagged" individual, to model the user's privacy preference we consider both sources of information (the region of focus and the surrounding context) and learn to accordingly weigh each of them.

\footnotetext{
${ }^{1}$ This scale is consistent with the scale used in prior related work [11]
} 
Our problem setting is non-trivial for a number of reasons. First, it is extremely hard to properly collect a large amount of labeled data from actual image owners, especially for shared and co-owned images. Thus an intuitive personalized model approach, for example based on a trained model for every single user, may not perform well. Second, considerations related to users' relationships must be accounted for. Users may have different degrees of confidence on other stakeholders' choices, depending on both sensitivity of content and on their relationship with these stakeholders [1,8]. For instance, a close friend may be trusted in sharing many photos related to a recent club event, whereas an acquaintance may not be trusted in sharing online photos of a dinner among colleagues.

Finally, to successfully leverage users' patterns and choices per the dimensions above (e.g. preferences, sensitivity of content and relationship among users) historical data of image stakeholders is desirable - both image-specific and even contextual data (e.g. social network habits). However, it is likely that only limited user historical data is available from social network platforms or end users. This leads to the well-known "cold start problem". It is known to be hard to deal with cold start issues, and we note the particular challenge of this issue in our work, where historical data is required for at least two separate users.

\section{TRAINING USER'S PRIVACY PREFERENCES}

We build a group-based model using information extracted for the image uploaders, who will maintain a more comprehensive view of the image content, and the tagged user(s), who will prioritize their own portion of the content. We then set a threshold to detect a conflict based on the estimated outputs of the two models.

\subsection{Model}

We assume there are $N_{u}$ uploaders and tagged user pairs, with each user pair $p$ made up of at least two user roles-uploader $u p$ and tagged user tag, involving $N_{p}$ images. Each uploader has a corresponding label list $\mathbf{b}_{\mathbf{p}_{\text {up }}}$ (denoting prior labeled images, and since we have multiple levels for the privacy label, we mapped these levels into values in $\{0,1 / 3,2 / 3,1\})$. Similarly, each tagged user is associated with a label list $\mathbf{b}_{\mathbf{p}_{\text {tag }}}$, indicating their privacy preferences for the images they previously labeled.

While privacy preferences are known to have a subjective component, we assume there are finite set of patterns for a user's privacy decisions[10, 12], even when they have different background and personal experience. This intuition allows us to manage the complexity of our problem: we group users by their privacy taste, and for each group, we build a classifier. Users' background (i.e. a set of finite demographic attributes) is also leveraged to infer the group belonging of a user. These groups need to be discovered through our learning mechanism (see Equation (4)). Here we assume there are $\mathrm{K}$ privacy groups ( $\mathrm{K}=6$ in our experiments).

We consider an image $i$ and one or more focused regions within it. We parameterize the probability of a given image $i$ provided by user pair $p$ being private to a user with role $\mathrm{r} \in\{u p, \operatorname{tag}\}$ (this user is denoted as $p_{r}$ for short), assuming he is in group $k$, as:

$$
\begin{aligned}
& P\left[C_{p_{r} i k}=1 \mid x_{p, i}\right]= \\
& \left.\sigma\left(W o_{k, r} * h\left(W h_{k, r} * x_{p, i, r}+b h_{k, r}\right)+b o_{k, r}\right)\right),
\end{aligned}
$$

where $C_{p_{r} i k}$ is the class label for the ith image for user $p_{r}$, who is in group $k . \sigma(\bullet)$ is the sigmoid activation function, $\mathrm{h}(\bullet)$ is the tanh activation function, $W h, b h$ are the hidden layer's parameters (weights and bias), Wo, ho is the output layer's parameters, $x_{p i, u p}$ is the whole image feature; $x_{p i, t a g}$ is the focus region feature.

For each image, assuming without loss of generality that there is only a single tagger, we assume two contributions to their privacy score, one from the whole image and one from the focus region. We assume that in each group according to the users' role within an image (uploader vs. tagger) and their relationship with the other image stakeholder, these two sources are treated differently.

Thus, we model the probability of the user focusing on the whole image as a function of their role and their relationship with the other stakeholder:

$$
P_{-} \text {focus }_{k, r}=\sigma\left(W r_{k, r} * r e l_{p i}+b r_{k, r}\right)
$$

where $W r, b r$ are parameters and $r e l_{p i}$ is the indicator vector for the relationship corresponding to the ith image labeled by user pair $p$. The final privacy score is:

$$
\begin{array}{r}
P\left[C_{p_{r} i k}=1 \mid \mathbf{X}_{p i}, \text { rel }_{p i}\right] \\
=P_{-} \text {focus }_{k, r} * P\left[C_{p_{r} i k}=1 \mid x_{p, i, u p}\right]+ \\
\left(1-P_{-} \text {focus }_{k, r_{p i}}\right) * P\left[C_{p_{r} i k}=1 \mid x_{p, i, t a g}\right],
\end{array}
$$

$\mathbf{x}_{p i}$ the union of $x_{p, i, u p}$ and $x_{p, i, t a g}$

The prior probability of a given user $p_{r}$ being in group $k$ is:

$$
\operatorname{prior}_{p_{r} k}=\frac{\exp \left(\beta_{k}^{t} \mathbf{y}_{p_{r}}+\beta_{k 0}\right)}{\sum_{k^{\prime}=1}^{K} \exp \left(\beta_{k^{\prime}}^{t} \mathbf{y}_{p_{r}}+\beta_{k 0^{\prime}}\right)},
$$

$y_{p_{r}}$ the user $p_{r}$ 's demographic information feature, $\beta_{k}$ and $\beta_{k 0}$ the weight vector and bias for group $k$.

Under an independent and identically distributed training data assumption, we express the pseudo incomplete data label posterior $\log$ likelihood function over all the training images of all users as:

$$
\begin{aligned}
& \sum_{p=1}^{N_{u}} \log \left(\sum _ { k = 1 } ^ { K } \left\{\text { prior }_{p_{u p} k} * \prod_{i=1}^{N_{p}}\left\{P\left[C_{p_{u p} i k}=1 \mid \mathrm{X}_{p i}, r e l_{p i}\right]\right)^{b_{p_{u p} i}}\right.\right. \\
& *\left(1-P\left[C_{p_{u p} i k}=1 \mid \mathbf{X}_{p i}, r e l_{p i}\right]\right)^{\left.\left.\left.1-b_{p_{u p} i}\right\}\right\}\right)+} \\
& \log \left(\sum _ { k = 1 } ^ { K } \left\{p _ { \text { tag } } * \prod _ { i = 1 } ^ { N _ { p } } \left\{P\left[C_{p_{\text {tag }} i k}=1 \mid \mathrm{X}_{p_{i}}, r e l_{p i}\right]^{b_{p_{\text {tag }} i}}\right.\right.\right. \\
& \left.\left.\left.*\left(1-P\left[C_{p_{\text {tag }} i k}=1 \mid \mathrm{X}_{\text {pi }}, r e l_{\text {pi }}\right]\right)^{1-b_{p_{\text {tag }} i}}\right\}\right\}\right) .
\end{aligned}
$$

\subsection{Optimization}

Since it is difficult to directly optimize the pseudo incomplete data $\log$ likelihood function from Equation (5), we use the Expectation Maximum (EM) algorithm. The pseudo complete data log-likelihood is: 


\begin{tabular}{|l||c|c|c|c|}
\hline Classifier & Overall Accuracy & TNR & TPR & F1-measure \\
\hline LRC & $52.53 \%$ & $62.02 \%$ & $43.03 \%$ & 0.508 \\
\hline LRR & $55.47 \%$ & $57.80 \%$ & $53.13 \%$ & 0.5536 \\
\hline MLP & $57.38 \%$ & $57.56 \%$ & $57.19 \%$ & 0.574 \\
\hline PMLP & $64.32 \%$ & $59.46 \%$ & $69.18 \%$ & 0.6395 \\
\hline MPC Model & $\mathbf{7 0 . 7 1 \%}$ & $\mathbf{6 7 . 8 9} \%$ & $\mathbf{7 3 . 4 8} \%$ & $\mathbf{0 . 7 0 5 5}$ \\
\hline
\end{tabular}

Table 1: Performance using multiclass label the second dataset is collected from Instagram [3]. Among these two image sources, we randomly select 3000 images and evenly split them into 100 subsets such that each set has 30 images. Each image is further labeled by two labelers to include a "bounding box" denoting a hypothetical stakeholder in the image (e.g. a face, an object or a living thing that would possibly be linked to someone other than the uploader). Each subset was assigned to two unique Crowdflower workers (upon proper ethical board approval), which are assigned as the (hypothetical) uploader and the user who is being tagged. Each labeler is first asked to provide their information (defined by 7 attributes, similar to [12]), and is assigned 15 images each. Each image is shown along with the bounding boxes denoting $\sum_{p=1}^{N_{u}}\left\{\sum_{k=1}^{K} V_{p_{u p} k}\left\{\log \left(\right.\right.\right.$ prior $\left._{p_{u p} k}\right)+\sum_{i=1}^{N_{p}}\left\{b_{p_{u p} i} \log \left(P\left[C_{p_{u p} i k}=1 \mid \mathbf{X}_{p i}, r e l_{p i}\right]\right)\right.$ $\left.\left.\left.+\left(1-b_{p_{u p} i}\right) \log \left(1-P\left[C_{p_{u p} i k}=1 \mid \mathbf{X}_{p i}, r e l_{p i}\right]\right)\right\}\right\}\right\}+$ $\left\{\sum_{k=1}^{K}\left\{V_{p_{\text {tag }} k}\left\{\log \left(\right.\right.\right.\right.$ prior $\left._{p_{\text {tag }} k}\right)+\sum_{i=1}^{N_{p}}\left\{b_{p_{\text {tag }} i} \log \left(P\left[C_{p_{\text {tag }} i k}=1 \mid \mathbf{X}_{\text {pi }}\right.\right.\right.$, rel $\left.\left.l_{\text {pi }}\right]\right)$ $+\left(1-b_{p_{\text {tag }} i}\right) \log \left(1-P\left[C_{p_{\text {tag }} i k}=1 \mid \mathrm{X}_{\text {pi }}\right.\right.$, rel $\left.\left.\left.\left.\left._{\text {pi }}\right]\right)\right\}\right\}\right\}$.

where $V_{p}$ is the latent variable indicate the group membership for user $p$. Specifically, $b_{\text {pri }}$ here is the ground truth label (values in $\{0$, $1 / 3,2 / 3,1\}$ ) for image $i$ labeled by user $p r$.

In the E-step, the expectation of the latent variable is calculated when given the observed data:

$$
\begin{aligned}
& E\left[V_{p_{r} k} \mid \mathbf{X}_{\mathbf{p}}, \mathbf{b}_{\mathbf{p}_{\mathbf{r}}}\right]=P\left[k \mid \mathbf{X}_{\mathbf{p}}, \mathbf{b}_{\mathbf{p}_{\mathbf{r}}}\right] \propto \\
& \exp \left(\log \left(\text { prior }_{p_{r} k}\right)+\sum_{i=1}^{N_{p}}\left\{b_{p_{r} i} \log P\left[C_{p_{r} i k}=1 \mid \mathbf{X}_{p i}, r e l_{p i}\right]\right.\right. \\
& \left.\left.+\left(1-b_{p_{r} i}\right) \log \left(1-P\left[C_{p_{r} i k}=1 \mid \mathbf{X}_{p i}, r e l_{p i}\right]\right)\right\}\right) .
\end{aligned}
$$

In the M-step, we fix the expected latent variables and maximize the expected value of the pseudo complete data log likelihood over the weight vector model parameters. In particular, since a closed form solution does not exist, we use a gradient descent based methodRMSProp[2], to optimize the parameters.

\subsection{Prediction}

Given that a new user $p$ has already labeled some images, we can compute $P\left[k \mid \mathbf{X}_{\mathbf{p}}, \mathbf{b}_{\mathbf{p}_{\mathbf{r}}}\right]$ according to the E-step equ. (7). For an image $i$, we can compute the a posterior privacy probability for user $p$ as:

$$
P\left[C_{p_{r} i} \mid \mathbf{X}_{\mathbf{p}}, \mathbf{b}_{\mathbf{p}_{\mathbf{r}}}\right]=\sum_{k=1}^{K} P\left[k \mid \mathbf{X}_{\mathbf{p}}, \mathbf{b}_{\mathbf{p}_{\mathbf{r}}}\right] P\left[C_{p_{r} i k}=1 \mid \mathbf{X}_{p i}\right] .
$$

Upon calculating two users' privacy probabilities seperately, we can compare the uploader and tagged user's results with an offset, which is chosen to balance the true positive rate (TPR) and true negative rate (TNR) of the validation set's result. If the tagged user's output is greater than the uploader's output plus the offset, we then say there is a conflict between them.

\section{EXPERIMENTAL EVALUATION}

\subsection{Datasets}

Our dataset is extracted from two existing online image datasets. The first set of images is originally obtained from [11], whereas the focus regions, with a predefined relationship (which varies for checked for quality; about $1 \%$ of the responses were removed due to poor performance (e.g. not completing tasks or not passing quality of the cases were noted as conflicts. In terms of demographics, $65.5 \%$ of the respondents are male, the average age is in the range of 25-34, and the majority respondent has a college degree (51.5\%). A plurality of the population is white (44.5\%), followed by Hispanic or Latino, other ethnicity, African American and Asian. Finally, a plurality of respondents access social networks daily(33.5\%) and post to social network sites frequently, about once every few days(58.5\%)

\subsection{Deep Learning for Feature Extraction}

Our image features are deep learning(DL) features, constructed as follows. We use the pre-trained eight-layer implementation of Convolutional Neural Networks implemented by Caffee [5], which is a benchmark standard for image classification and object detection tasks. The first five layers of this network extract features by convolution with a set of image filters. Because we are not interested in object identification, we disregard the 8th layer and treat the 4096-dimensional output of the 7th layer of the network as a feature vector, describing high-level features of each image or image patch. Note that prior to feeding an image or an image patch into the Deep Learning Network, it is re-sized to $224^{\star} 224$. In this way, a patch can be represented as a 4096 dimension DL feature vector regardless of its size.

\subsection{Baselines and Experimental Settings}

We tested several standard baseline algorithms to assess the performance of our proposed algorithm.

Logistic Regression(LR) Two simple LR based approaches are used as baselines. The first LR baseline is trained for binary conflict classification using the concatenation of all available features of a user pair. The second LR model is trained to infer each user's decision. Here, we name them LRC and LRR correspondingly, 11 regulation was used for both approaches.

Multilayer Perceptron (MLP) Our most sophisticated baseline classifier relies on a Convolutional Neural Network architecture. Since the image features are extracted from a CNN framework per Section 3.2, we adopt transfer learning to build an MLP network with a fully connected layer and a decision layer, and let the model predict a user's privacy preference. We first feed a user's corresponding image features into a hidden layer of 50 rectified linear checks). In total, 100 valid user pair responses were collected. $8.87 \%$ 
units (ReLU). Then we concatenate these units' outputs with the user's demographic features, relationship features and role into the hidden layer. Lastly, a final sigmoid unit is used as the output layer. Again, we used ADAM to minimize the mean squared error.

For even closer similarity with our own work, we also adopted a more personalized way to train the MLP, referred to as PMLP. We first train an MLP using the method above; then for every test user, we adapt (customize) the parameters based on the first 15 (labeled) images, using a small number of iterations.

We used 10-fold nested cross validation for our experiments. More precisely, the dataset was split into 10 folds, 9 used for training and 1 for testing. Among the training folds, for every subset as we described before, we used the first 27 images for training, with the remaining 3 for validation. For our test set, the first 15 images were treated as the user's decision history, with the remaining half used for testing. Since the model's parameter set is relative large compared to the data size, to avoid overfitting we used only 10 EM iterations, and in the M-step only 10 gradient steps. We choose the model with the best validation accuracy for testing.

\subsection{Results}

As reported in Table 1, LRC achieved poor performance, with TPR and TNR of only $62.02 \%$ and $43.03 \%$. We attribute this to the fact that although we use L1 regularization to avoid overfitting, the feature dimension is still quite high (8266) compared with the training data size (2580). Also, in this experiment we directly predict a conflict label: i) this loses the individual user privacy label information; ii) the conflict label is highly class-imbalanced. We expect improvement by training the model based on the original labels directly, and then inferring the conflict event. Accordingly, we tested the modified LRR model. In the testing phase, we first separately predict two users' privacy preference; then compare the two outputs with the same approach as introduced in Section 2.3. This slightly improves the performance compared with LRC.

Our richer baseline, MLP, improves the overall accuracy by about $2 \%$ over LRR. However, both LRR and MLP did not provide strong results. As expected, PMLP performs the best over all baselines, since it is designed to place more focus on the stakeholders' subjective privacy preferences. However, possibly due to limited training data, PMLP only achieved an accuracy of $64.32 \%$.

Our proposed model outperformed all the baselines significantly; it reached a TNR of $67.89 \%$ and TPR of $73.48 \%$. This group based approach works better for our problem as it combines semi-personalized analysis of users' content while borrowing statistical strength across users in the same group to deal with the limited training data problem. We also compute the Precision-Recall Curve, as shown in Figure 2. Our proposed algorithm reached the highest area under curve(AUC) of 0.8097 , while MLP and PMLP only obtained an AUC of 0.6043 and 0.769 , respectively.

\section{DISCUSSION AND CONCLUSION}

This work provides a first group-based approach toward addressing automated conflict detection in online images. Despite some promising initial results, much is left to be done to achieve a comprehensive solution. Below we briefly discuss some open issues.

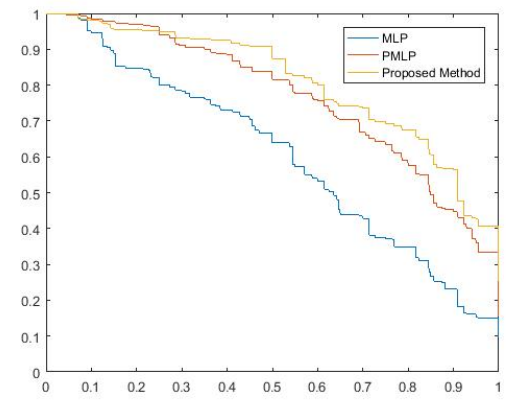

Figure 2: Precision-Recall Curve

Our results are thus far based on the simpler case of one uploader and one stakeholder. Clearly, this may not always be the case, as more users are present in a single piece of content. While the model is designed to support any number of stakeholders, we are yet to validate this case empirically. While our use of four privacy label values is a de-facto standard, a more fine grained representation of users' privacy preferences (e.g. by audience groups) could help improve generalizability of our approach. Due to space limitations we have so far reported only the main findings from our model. It would be important to expand the empirical evaluation and give an extensive treatment of cases where users have no historical data. Acknowledgments Work from Dr Squicciarini and Haoti Zhong was partially funded by National Science Foundation under grant 1421776 and 1453080.

\section{REFERENCES}

[1] F. Adu-Oppong, C. K. Gardiner, A. Kapadia, and P. P. Tsang. Social circles: Tackling privacy in social networks. In Symposium on Usable Privacy and Security (SOUPS), 2008.

[2] G. Hinton, N. Srivastava, and K. Swersky. Neural networks for machine learning lecture 6a overview of mini-batch gradient descent.

[3] H. Hosseinmardi, R. I. Rafiq, R. Han, Q. Lv, and S. Mishra. Prediction of cyberbullying incidents in a media-based social network. In ASONAM, pages 186-192. IEEE, 2016.

[4] H. Hu, G.-J. Ahn, and J. Jorgensen. Detecting and resolving privacy conflicts for collaborative data sharing in online social networks. In Proceedings of the 27th Annual Computer Security Applications Conference, pages 103-112. ACM, 2011.

[5] Y. Jia, E. Shelhamer, J. Donahue, S. Karayev, J. Long, R. Girshick, S. Guadarrama, and T. Darrell. Caffe: Convolutional architecture for fast feature embedding. In ACM International Conference on Multimedia, pages 675-678. ACM, 2014.

[6] D. Kekulluoglu, N. Kokciyan, and P. Yolum. Preserving privacy as social responsibility in online social networks. ACM Transactions on Internet Technology (TOIT), 18(4):42, 2018.

[7] S. Rajtmajer, A. Squicciarini, J. M. Such, J. Semonsen, and A. Belmonte. An ultimatum game model for the evolution of privacy in jointly managed content. In International Conference on Decision and Game Theory for Security, pages 112-130. Springer, 2017.

[8] E. Spyromitros-Xioufis, S. Papadopoulos, A. Popescu, and Y. Kompatsiaris. Personalized privacy-aware image classification. In Proceedings of the 2016 ACM on International Conference on Multimedia Retrieval, pages 71-78. ACM, 2016.

[9] J. M. Such and N. Criado. Resolving multi-party privacy conflicts in social media. IEEE Transactions on Knowledge and Data Engineering, 28(7):1851-1863, 2016.

[10] Y. Wang, G. Norice, and L. F. Cranor. Who is concerned about what? a study of american, chinese and indian users' privacy concerns on social network sites. In International Conference on Trust and Trustworthy Computing, pages 146-153. Springer, 2011.

[11] S. Zerr, S. Siersdorfer, and J. Hare. Picalert!: a system for privacy-aware image classification and retrieval. In Proceedings of the 21st ACM international conference on Information and knowledge management, pages 2710-2712. ACM, 2012.

[12] H. Zhong, A. C. Squicciarini, D. J. Miller, and C. Caragea. A group-based personalized model for image privacy classification and labeling. In Twenty-Sixth International foint Conference on Artificial Intelligence, pages 3952-3958, 2017. 